\title{
THREE POINT ARCWISE CONVEXITY
}

\section{F. A. VALENTINE}

Let $S$ be a set in a two dimensional Euclidean space $E_{2}$. Such a set $S$ is said to be arcwise convex [5] if each pair of its points can be joined by a convex arc lying in $S$. A convex arc is, by definition, an arc which is contained in the boundary of a plane convex set. In two previous papers, $[5 ; 6]$, the author studied certain properties of closed arcwise convex sets. It is the purpose of this paper to study an interesting class of sets which satisfy the three point arcwise convexity property, defined as follows:

DEFINITION 1. A set $S \subset E_{2}$ is said to have the three point arcwise convexity property if each triple of points $x \in S, y \in S, z \in S$ is contained in a convex arc belonging to $S$.

It should be observed that the above property implies there exists a convex arc in $S$ having two of the three points $x, y, z$ as end points and the third point in its interior.

Definition 2. A convex curve, as distinguished from a convex arc, is a closed connected portion of the boundary of a plane convex set.

A convex curve may have two, one, or no end points, and it may be bounded or unbounded.

The following theorem characterizes the closed sets in $E_{2}$ which have the three point arcwise convexity property.

THEOREM 1. Let $S$ be a closed set in $E_{2}$ which has at least three points. Then $S$ has the three point arcwise convexity property if and only if it satisfies at least one of the following three conditions.

1. It is a closed convex set.

2. It is a convex curve.

3. It is a closed convex set except for one bounded convex hole, that is, it is obtained by deleting from a closed convex set a bounded open convex subset.

In order to prove the necessity of Conditions $1-3$, we shall establish four lemmas. In each of them it is assumed that $S$ is a closed set which has at least three points, and which has the three point arcwise convexity property.

LEMмA 1. If $x, y, z$ are three collinear points of $S$, with $y$ between $x$ and $z$, then at least one of the closed segments $x y$ or $y z$ belongs to $S$.

Presented to the Society, November 27, 1954; received by the editors September $28,1954$. 
Proof. In this and later proofs we use the following notation.

Notation. The boundary of a set $C$ is denoted by $B(C)$. A convex arc in $S$ containing the points $x, y$, and $z$ and having two of them as end points is denoted by $C(x, y, z)$.

To prove Lemma 1 , let $H$ be the convex hull of $C(x, y, z)+x z$. Since $C(x, y, z)$ is a convex arc, we have $C \equiv C(x, y, z) \subset B(H)$. This implies that if $z$ is an end point of $C$, then $x y \subset S$. On the other hand, if $z$ is not an end point of $C$, then $y z \subset S$.

Lemma 2. Suppose a component $K$ of the complement of $S$ exists which is unbounded, and which has a cross-cut [a cross-cut of $K$ is a closed segment $x y$ with $x y-x-y \subset K$ and with $x \in B(K), y \in B(K)]$. Then $S=B(K)$, and $B(K)$ is a convex curve.

Proof. Since $K$ is a component of the complement of a closed connected set, it is known $[3$, pp. 108, 117] that $K-x y$ is the sum of two mutually exclusive open connected sets. One of these is bounded, since $S$ has the three point arcwise convexity property. We denote this bounded component by $K_{1}$. We shall prove that $\bar{K}_{1}$ (the closure of $K_{1}$ ) is a convex set. It follows from Lemma 1 immediately that $K_{1}$ lies entirely in one of the two open half-planes determined by the line $L(x, y)$ containing $x y$. Denote this open half-plane by $R$. Moreover, let $L(x)$ be a closed ray with $x$ as its end point. If $L(x) \cdot K_{1} \neq 0$, Lemma 1 implies $L(x) \cdot K_{1}$ is an interval with end points $z$ and $p$, where $p \neq x$, and $x z \subset x p$. If $z \neq x$, then the convex arc $C(x, y, z)$ would contain $z$ in the interior of its convex hull, since $K$ is unbounded, and since $L(x) \cdot K_{1} \neq 0$. However, this contradicts the convexity of the arc $C(x, y, z)$, so that we must have $L(x) \cdot B\left(K_{1}\right)=x+p$ when $L(x) \cdot K_{1} \neq 0$. Similarly, $L(y) \cdot B\left(K_{1}\right)=y+s$, if $L(y) \cdot K_{1} \neq 0$. Now, choose any pair of points $u \in K_{1}, v \in K_{1}$. If the line $L(u, v)$ intersects the interior of the segment $x y$, then the lines $L(x, u), L(y, u), L(x, v)$, $L(y, v)$ determine a quadrilateral $Q$ having its sides in $K_{1}$, and having $u v$ as a diagonal. This, together with the connectness of $B\left(K_{1}\right)$, implies that $Q \subset K_{1}$. Hence, $u v \subset K_{1}$. Secondly, if $L(u, v) \cdot x y=0$, then Lemma 1 implies that $L(u, v) \cdot K_{1}$ is connected. Hence, we have shown that $K_{1}$ is convex. Thus, $\bar{K}_{1}$ is also convex.

Next, we prove that $S \cdot R=B\left(K_{1}\right)-x y$, where $R$ is the open halfplane defined above. First, suppose there exists a point $q \in S \cdot R-\bar{K}_{1}$ such that $L(x, q) \cdot K_{1} \neq 0$. Then, as proved above, $L(x, q) \cdot B\left(K_{1}\right)$ $=x+p$, and $p$ is between $x$ and $q$ on the line $L(x, q)$. The line $L(x, q)$ determines an open half-plane $R_{1}$ which does not contain $y$. Choose a ray $L(q)$ with end point $q$ which is in $\bar{R}_{1}$, and which intersects $K_{1} \cdot R_{1}$. Since $\bar{K}_{1}$ is convex, and since $q \notin \bar{K}_{1}$, we have $L(q) \cdot B\left(K_{1}\right)=u+v$, where $u \in S \cdot R_{1}, v \in S \cdot R_{1}$, and where $u$ is between $q$ and $v$ on $L(q)$. 
Since $K$ is unbounded, a convex $\operatorname{arc} C(x, u, q) \subset S$ is contained in $\bar{R}_{1}$. Since $L(q) \cdot K_{1} \neq 0$, and since the convex hull $K^{*}$ of $C(x, u, q)+x q$ contains $\bar{K}_{1} \cdot \bar{R}_{1}$, the point $u$ is an interior point of $K^{*}$, and this is impossible since $C(x, u, q)$ is a convex arc. Hence, we have a contradiction, and therefore no point $q \in S \cdot R-\bar{K}_{1}$ exists for which $L(x, q)$ - $K_{1} \neq 0$. Similarly no point $q \in S \cdot R-\bar{K}_{1}$ exists for which $L(y, q)$ $\cdot K_{1} \neq 0$. Finally, if a point $q \in S \cdot \bar{R}-\bar{K}_{1}$ exists such that $x q \cdot K_{1}=0$, $y q \cdot K_{1}=0$, then a convex arc $C(x, y, q) \subset S$ bounds a convex set containing $K_{1}$ in its interior. However, in this case, there would exist a ray $L(u)$ with $u=x$ or $u=y$ such that $L(u) \cdot K_{1} \neq 0$, and such that $L(u) \cdot S \neq L(u) \cdot B\left(K_{1}\right)$. This contradicts the previously proven fact that when $L(u) \cdot K_{1} \neq 0$, then $L(u) \cdot S$ consists of two points of $S$. Thus $S \cdot R=B\left(K_{1}\right)-x y \equiv C$, a convex arc of $S$. This is true for each crosscut of $K$.

Now, to complete the proof of Lemma 2, since $K$ has a cross-cut, the above implies that $B(K) \cdot S$ has a nonempty convex arc $C$ as a subset. Let $C^{*}$ be the maximal closed convex curve of $B(K) \cdot S$ which contains $C$. We shall prove that $C^{*}=B(K)=S$. To do this, first suppose $C^{*}$ has two end points, $x$ and $y$, and let $R$ be the open half-plane containing $C^{*}$ as defined above by means of $L(x, y)$. Suppose a point $z \in S$ exists which is not in $\bar{R}$. Since $S \cdot \bar{R}=C^{*}$, a convex $\operatorname{arc} C(x, y, z)$ $C S$ containing $x, y$ and $z$ would contradict the fact that $C^{*}$ is maximal. Hence, in this case, $S \cdot \bar{R}=C^{*}=S$. Similarly, if $C^{*}$ has one or no end points, Lemma 1 and the maximality of $C^{*}$ imply that $C^{*}=S$, a convex curve. This completes the proof of Lemma 2 .

Lemma 3. The complement of the set $S$ has at most one bounded component, and it is convex.

Proof. This is an immediate consequence of Lemma 1.

The following elementary lemma is known, and it is included for sake of completeness.

LEMMA 4. If an open set $K$ has no cross-cuts, then it is the complement of a convex set.

Proof. Let $x$ and $y$ be two points not in $K$. If $x y \cdot K \neq 0$, then it is clear that $K$ has a cross-cut which is a subinterval of $x y$. Hence $K$ is the complement of a convex set.

Proof of Theorem 1. Necessity. If a set $S$ satisfies the conditions of Lemma 2, then it satisfies Condition 2 of the theorem. If $K$ satisfies the conditions of Lemma 4 , then $S$ satisfies either Conditions 1 or 3 of the theorem. If the complement of $S$ is bounded, Lemma $3 \mathrm{im}$ plies that $S$ satisfies Condition 3. Thus Theorem 1 is a direct consequence of Lemmas 1-4. 
Sufficiency. If $S$ satisfies Condition 1 or 2 of Theorem 1, it obviously satisfies the three point convexity property. Suppose $S$ then satisfies Condition 3 , and let $x, y, z$ be any three points in $S$, and let $K$ be the bounded component of the complement. Let $H$ be. the convex hull of $\bar{K}+x+y+z$. Since $S+K$ is a convex set, the boundary $B(H)$ is in $S$. If $x \in B(H), y \in B(H), z \in B(H)$ then clearly $x, y$, and $z$ are contained in a convex arc of $S$. If two of the points, $x, y$ and $z$, say $x$ and $y$, are in $B(H)$, and if the third is interior to $H$, then an arc of $B(H)$ joining $x$ and $y$ and a line segment in $S$ joining $z$ to $x$ or $z$ to $y$ (one of these must exist) again provide the desired convex arc. Thus $S$ satisfies the three point arcwise convexity property. This completes the proof of Theorem 1.

Otto Haupt in a paper titled Über eine Kennzeichnung der Kugel [2], studied sets $S \subset E_{n}$ such that for each triple of points in $S$ there exists a circular arc or a segment in $S$ which contains the three points. He calls such sets "Kreiskonvex." If $S$ is a compact kreiskonvex set containing interior points, Haupt proves that $S$ is a sphere. It is interesting to note that if in Haupt's theorem, we replace circular arcs by convex arcs, and if each three collinear points of $S$ are contained in a segment of $S$, then Theorem 1 implies that $S$ is a convex set. These last results are related to a result of G. Aumann [1]. "If each plane section of a compact set $S$ is a simply-connected continuum, then $S$ is convex."

E. G. Straus and F. A. Valentine [4] have investigated sets which have the "three-point linear convexity property." Such a set $S$ is one for which each of its triple of collinear points is contained in a segment of $S$. They have characterized the connected closed sets $S \subset E_{2}$ having this property, and the results will be prepared for possible publication.

\section{BIBLIOGRAPHY}

1. George Aumann, On a topological characterization of compact convex point sets, Ann. of Math. vol. 37 (1936) pp. 443-447.

2. Otto Haupt, Über eine Kennzeichnung der Kugel, Jber. Deutschen Math. Verein. vol. 50 (1940) pp. 113-120.

3. M. H. A. Newman, Elements of the topology of plane sets of points, Cambridge University Press, 1939.

4. E. G. Straus and F. A. Valentine, Three point convexity properties, (Unpublished).

5. F. A. Valentine, Arcwise convex sets, Proc. Amer. Math. Soc. vol. 2 (1951) pp. $159-165$.

6. - A characterization of simply connected closed arcwise convex sets, Proc. Amer. Math. Soc. vol. 2 (1951) pp. 778-780.

University of California, Los Angeles 